\title{
The speed of Muller's ratchet with background selection, and the degeneration of Y chromosomes
}

\author{
ISABEL GORDO* AND BRIAN CHARLESWORTH \\ Institute of Cell, Animal and Population Biology, University of Edinburgh, Ashworth Laboratories, Kings Buildings, West Mains Road, \\ Edinburgh EH9 3JT, UK
}

(Received 23 October 2000 and in revised form 5 February and 18 April 2001)

\begin{abstract}
Summary
The rate of accumulation of deleterious mutations by Muller's ratchet is investigated in large asexual haploid populations, for a range of parameters with potential biological relevance. The rate of this process is studied by considering a very simple model in which mutations can have two types of effect: either strongly deleterious or mildly deleterious. It is shown that the rate of accumulation of mildly deleterious mutations can be greatly increased by the presence of strongly deleterious mutations, and that this can be predicted from the associated reduction in effective population size (the background selection effect). We also examine the rate of the ratchet when there are two classes of mutation of similar but unequal effects on fitness. The accuracy of analytical approximations for the rate of this process is analysed. Its possible role in causing the degeneration of $\mathrm{Y}$ and neo-Y chromosomes is discussed in the light of our present knowledge of deleterious mutation rates and selection coefficients.
\end{abstract}

\section{Introduction}

Deleterious alleles are continuously arising in populations by mutation pressure, and are eliminated by selection (Crow, 1993). In addition to mutation and selection, the presence or absence of recombination in a population has a very strong impact on the dynamics of the deleterious mutations which it contains. With free recombination, deleterious mutations can be eliminated effectively but, in the absence of recombination, the efficacy of selection is reduced and deleterious mutations can accumulate in finite populations (Fisher, 1930, p. 122; Muller, 1964; Hill \& Robertson, 1966; Felsenstein, 1974). For deleterious mutations whose effect is much greater than the inverse of the population size, back-mutation is negligible, and the accumulation is effectively irreversible. This is the process known as Muller's ratchet (Muller, 1964; Felsenstein, 1974), whereby the mean number of deleterious mutations increases indefinitely at a constant mean rate in a finite nonrecombining population, due to the repetitive loss of

\footnotetext{
* Corresponding author. Tel: +44 (0) 13165086 24. Fax: +44 (0) 131 6506564. e-mail: i.gordo@ed.ac.uk
}

the least mutated classes of individuals. The rate of this process, measured by the inverse of the mean time to loss of the least-loaded class, is known to be an exponentially increasing function of the mutation rate, and a decreasing function of the population size and the fitness effects of the mutations (Haigh, 1978).

If mutations cause sufficiently large deleterious effects, the expected time for them to accumulate in large populations is so long that they effectively do not accumulate (Crow \& Kimura, 1970, chap. 9). An approximate condition on the mutation rate $(u)$, population size $(N)$ and selection coefficient $(s)$ for the ratchet not to operate in a reasonable time scale is given by $N s \exp (-u / s) \gg 15$ (Gordo \& Charlesworth, $2000 a$ ). Such mutations are kept indefinitely at frequencies close to those expected under equilibrium between mutation and selection. However, their presence is known to interfere with the dynamics of linked neutral or very weakly selected mutations (those for which $N|s| \sim O(1)$ ), and even of mutations with much larger effects (Fisher, 1930, p. 122; B. Charlesworth et al., 1993; Charlesworth, 1994; Peck, 1994; Barton, 1995; Stephan et al., 1999). Their presence affects both nucleotide site diversity and fixation probabilities at weakly selected linked sites 
(Charlesworth, 1994; Stephan et al., 1999), namely decreasing diversity, decreasing the probability of fixation of advantageous mutations, and increasing the probability of fixation of deleterious ones. In large populations with no recombination, this process can be quantified as though the effective population size is reduced approximately by a fraction $f_{0}$, the frequency of individuals free of deleterious mutations. This is the process called background selection (B. Charlesworth et al., 1993). The degeneration of large non-recombining portions of the genome such as the $\mathrm{Y}$ chromosome may be caused by the operation of Muller's ratchet (Charlesworth, 1978) and/or the fixation of weak deleterious mutations due to background selection (Charlesworth, 1996). Because both strongly and mildly deleterious mutations probably occur in natural populations (Keightley \& EyreWalker, 1999), it is important to quantify the effect of their simultaneous presence.

In this paper, we analyse the rate of accumulation due to the ratchet, using a simple model to study the effect of strongly deleterious mutations of effect $s_{\mathrm{b}}$ (those that do not accumulate and can cause background selection) on the rate of accumulation of mildly deleterious mutations of effect $s_{\mathrm{s}}$ (those that allow Muller's ratchet to operate) in haploid asexual populations. We show that the rate of accumulation can be greatly increased by the presence of strongly deleterious mutations, and that the effect of the latter is generally to reduce the effective population size as predicted by the theory of background selection (B. Charlesworth et al., 1993). Two approximations are proposed, which depend on the relative values of the parameters, to calculate the rate of accumulation of mildly deleterious mutations in this simple model. We conduct simulation-based studies for the more complex case where both types of deleterious mutations can accumulate, and compare this with the simple case of a population subject to just one type of mutation, whose effect is given by the harmonic mean of the effects in the two-type mutation model. As in the case of mutations with constant effects (Higgs \& Woodcock, 1995; Charlesworth \& Charlesworth, 1997; Bergstrom \& Pritchard, 1998), in this simple model the rate of accumulation is equal to the rate of fixation of deleterious mutations. The more realistic case of an arbitrary distribution of selection coefficients remains to be explored.

\section{Theoretical considerations}

(i) Muller's ratchet

Consider first an effectively infinite, non-recombining haploid population, subject to deleterious mutations whose effect on fitness is $s_{\mathrm{s}}$, and which occur according to a Poisson distribution with mean $u_{\mathrm{s}}$. With multi- plicative fitnesses, at mutation-selection balance, the expected frequency of individuals with $i$ mutations is

$p_{i}=\left(\frac{u_{\mathrm{s}}}{s_{\mathrm{s}}}\right)^{i} \frac{\exp \left(-u_{\mathrm{s}} / s_{\mathrm{s}}\right)}{i !}$,

i.e. a Poisson distribution with mean $\Theta_{\mathrm{s}}=u_{\mathrm{s}} / s_{\mathrm{s}}$ (Kimura \& Maruyama, 1966; Haigh, 1978). In particular, the size of the class of individuals free of deleterious mutations, usually called the least-loaded class, is $n_{0}=N \exp \left(-\Theta_{\mathrm{s}}\right)$. The operation of Muller's ratchet means that, in a finite population, this class may be lost by drift and replaced by the next leastloaded class, which will then become the new leastloaded class. This new class may also be lost, just like the previous one, and a ratchet-like process takes place at a stochastically constant rate (Haigh, 1978). When $n_{0}>1$, the quantification of the speed of this process has been treated as a one-dimensional diffusion process, in which the expected mean time to absorption of the least-loaded class is calculated under different approximations for the drift coefficient of the diffusion process (Stephan et al., 1993; Charlesworth \& Charlesworth, 1997; Gordo \& Charlesworth, $2000 a, b)$, or by dividing the process into two phases (Stephan et al., 1993). When $n_{0}<1$, its quantification can be treated by approximation of the moments of the distribution of the number of mutations (Gessler, 1995).

\section{(ii) Muller's ratchet with background selection}

Consider a very large, effectively infinite, haploid population, which does not recombine and is subject to deleterious mutations with two kinds of effect: $s_{\mathrm{s}}$ and $s_{\mathrm{b}}$ (with $s_{\mathrm{b}} \gg s_{\mathrm{s}}$ ). Let the fitness of an individual carrying $k$ mutations with effect $s_{\mathrm{s}}$ and $n$ mutations with effect $s_{\mathrm{b}}$ be $\left(1-s_{\mathrm{s}}\right)^{k}\left(1-s_{\mathrm{b}}\right)^{n}$. Let $p_{n k}$ be the frequency of individuals carrying $k$ mutations with effect $s_{\mathrm{s}}$ and $n$ mutations with effect $s_{\mathrm{b}}$. The recursion for $p_{n k}$ is

$$
\begin{aligned}
\bar{w} p_{n k}(t+1)=\sum_{i=0}^{n} \sum_{j=0}^{k} p_{i j}(t)\left(1-s_{\mathrm{b}}\right)^{i}\left(1-s_{\mathrm{s}}\right)^{j} \\
\times \frac{u_{\mathrm{b}}^{n-i}}{(n-i) !} \mathrm{e}^{-u_{\mathrm{b}}} \frac{u_{\mathrm{s}}^{k-j}}{(k-j) !} \mathrm{e}^{-u_{\mathrm{s}},}
\end{aligned}
$$

where $u_{\mathrm{b}}$ and $u_{\mathrm{s}}$ are the mutation rates for mutations with effect $s_{\mathrm{b}}$ and $s_{\mathrm{s}}$, respectively, and $\bar{w}$ is the population mean fitness.

Because new mutations are assumed to be Poissondistributed, the recursion for $p_{00}$ implies that the mean fitness at equilibrium (cf. Kimura \& Maruyama, 1966) is

$\bar{w}=\exp (-u)=\exp \left(-\left(u_{\mathrm{b}}+u_{\mathrm{s}}\right)\right)$. 
By substitution, it is easy to see that an equilibrium solution is

$p_{i j}=\frac{\Theta_{\mathrm{b}}^{i}}{i !} \mathrm{e}^{-\Theta_{\mathrm{b}}} \frac{\Theta_{\mathrm{s}}^{j}}{j !} \mathrm{e}^{-\Theta_{\mathrm{s}}}$,

where $\Theta_{\mathrm{b}}=u_{\mathrm{b}} / s_{\mathrm{b}}$ and $\Theta_{\mathrm{s}}=u_{\mathrm{s}} / s_{\mathrm{s}}$ (Johnson, 1999). Although the stability of this equilibrium solution has not been formally established, simulations suggest that it is a stable solution, when $N \rightarrow \infty$.

The probability of having $n=i+j$ mutations is given by the convolution of two Poisson distributions, which is a Poisson distribution with mean $\Theta_{b}+\Theta_{s}$. At equilibrium the number of individuals free of mutations is thus

$n_{00}=N \mathrm{e}^{-\Theta_{\mathrm{b}}} \mathrm{e}^{-\Theta_{\mathrm{s}}}$.

Assume that $N$ is large and that $u_{\mathrm{b}} / s_{\mathrm{b}}$ is small, so that strongly deleterious mutations are maintained close to equilibrium (this is expected to occur if $N s_{\mathrm{b}} \exp \left(-u_{\mathrm{b}} / s_{\mathrm{b}}\right) \gg 15$ : Gordo \& Charlesworth, $2000 a$ ). This implies that the fraction of the population that is free of these mutations is given by

$f_{0 \mathrm{~b}}=\exp \left(-u_{\mathrm{b}} / s_{\mathrm{b}}\right)$.

The background selection principle (B. Charlesworth et al., 1993) implies that neutral or weakly selected mutations can spread to fixation only if they arise on a background free of strongly deleterious ones (Fisher, 1930, Charlesworth, 1994; Peck, 1994; Barton, 1995). This suggests that mutation accumulation by the ratchet will only occur by an increase in the number of mildly deleterious mutations in a background free of strongly selected mutations, when these are being held at deterministic equilibrium.

This will affect the rate of the ratchet for mildly deleterious mutations as follows. Let $x$ denote the frequency of the least-loaded class, whose initial frequency is $x_{0}=\exp \left(-u_{\mathrm{s}} / s_{\mathrm{s}}\right) \exp \left(-u_{\mathrm{b}} / s_{\mathrm{b}}\right)$. We will determine the expected time until absorption (at $x$ $=0$ ) using a diffusion equation as previously (Stephan et al., 1993; Charlesworth \& Charlesworth, 1997; Gordo \& Charlesworth, $2000 a, b$ ). Call $y$ the frequency of the class that is free of mildly deleterious mutations, so that $x=y \exp \left(-u_{\mathrm{b}} / s_{\mathrm{b}}\right)$. Because, by assumption, the frequency of the class free of strong mutations does not change, for $y \leqslant 1$ the relevant diffusion coefficient, approximated by the variance due to binomial sampling in a Wright-Fisher population, is

$b(y) \approx \frac{y}{N \exp \left(-u_{\mathrm{b}} / s_{\mathrm{b}}\right)}$

and, using the same assumptions as in Gordo \& Charlesworth (2000a), the drift coefficient is approximated by

$a(y) \approx 0.6 s_{\mathrm{s}}\left(1-y / f_{0 \mathrm{~s}}\right) y$, where $f_{0 \mathrm{~s}}=\exp \left(-u_{\mathrm{s}} / s_{\mathrm{s}}\right)$. This means that the mean time to absorption (Ewens, 1979, chap. 4), i.e. the mean time for a click of the ratchet, is the same as in the case of a population subject to mutations with just one selection coefficient (of value $s_{\mathrm{s}}$ ) occurring at a rate $u_{\mathrm{s}}$, but whose size is $N f_{0 \mathrm{~b}}$, i.e. the size of the leastloaded class is discounted by a factor equal to the frequency of the least-loaded class with respect to the strongly selected mutations.

It is clear that, if the frequency of the class free of strong deleterious mutations is not constant, then a one-dimensional diffusion equation will not be adequate, and stochastic changes in the frequency of the strong class have to be taken into account, as well as the covariance between the frequency of the strong and mildly deleterious mutations, making the analysis extremely difficult.

We must also account for the time taken for the value of the least-loaded class after a click to reach a value close to that expected at mutation-selection equilibrium (Haigh, 1978; Stephan et al., 1993). This can be approximated deterministically as in equation (1) of Gordo \& Charlesworth $(2000 \mathrm{~b})$. The total time for a click of Muller's ratchet will then be given by

$$
\begin{aligned}
& T\left(N, u_{\mathrm{s}}, s_{\mathrm{s}}, u_{\mathrm{b}}, s_{\mathrm{b}}\right)=\frac{1}{s_{\mathrm{s}}}\left(1-1.6 \frac{s_{\mathrm{s}}}{u_{\mathrm{s}}}\right)+\int_{0}^{f_{0 \mathrm{~s}}} \frac{2 N f_{0 \mathrm{~b}}}{x G(x)} \\
& \left\{\int_{0}^{x} G\left(x^{\prime}\right) \mathrm{d} x^{\prime}\right\} \mathrm{d} x+\int_{f_{0 \mathrm{~s}}}^{1} \frac{2 N f_{0 \mathrm{~b}}}{x G(x)}\left\{\int_{0}^{f_{0 \mathrm{~s}}} G\left(x^{\prime}\right) \mathrm{d} x^{\prime}\right\} \mathrm{d} x,
\end{aligned}
$$

where

$G\left(x^{\prime}\right)=\exp \left(\frac{2 N f_{0 \mathrm{~b}}}{f_{0 \mathrm{~s}}} 0.6 s_{\mathrm{s}} x^{\prime}\left(\frac{x^{\prime}}{2}-f_{0 \mathrm{~s}}\right)\right)$.

The first term represents the approximate time to approach a value close to the equilibrium and the other two terms represent the mean time to absorption of the stochastic process with initial frequency $f_{0 \mathrm{~s}}$.

\section{Simulation methods}

For a given population size $(N)$, genomic mutation rate to deleterious mutations $(u)$ and selection coefficients against each mutation $\left(s_{\mathrm{s}}\right.$ or $\left.s_{\mathrm{b}}\right)$, haploid asexual populations were simulated, starting with all individuals initially free of mutations. Assuming that the sequence of events is mutation, reproduction and selection (Gordo \& Charlesworth, 2000a), populations were then run for an initial period of generations so that they could approach an equilibrium between mutation and selection (i.e. the mean fitness of the population reaches the value $\exp (-u)$ ). An additional time until a click of the ratchet for the mutations of small effect was observed was allowed. After this initial period, populations were run for more than 
2000 generations, and up to 100000 generations, depending on the speed at which mutations accumulated. Every generation, the number of mildly selected mutations and strongly selected mutations in every individual were counted, and the number of individuals with the least number of mutations (leastloaded class) recorded. If, at a given generation, the number of mutations in the least-loaded class increases, the ratchet has clicked.

To form a new generation, individuals are sampled randomly from the previous generation, then subjected to the occurrence of mutations sampled from a Poisson distribution that can have either an effect $s_{\mathrm{s}}$ (mildly deleterious mutations) or $s_{\mathrm{b}}$ (strongly deleterious mutations) on fitness, and assigned probabilities of survival $\left(1-s_{\mathrm{s}}\right)^{k}\left(1-s_{\mathrm{b}}\right)^{n}$, where $k$ is the number of mutations with effect $s_{\mathrm{s}}$ and $n$ is the number of mutations with effect $s_{\mathrm{b}}$ carried by an individual. A new generation of $N$ individuals is constructed by comparing the probability of survival of each individual with a pseudo-random number drawn from a uniform distribution in the interval $[0,1]$. Each run was repeated five times and the average rate of accumulation of mutations, measured by the average time to loss of the least-loaded class, was computed.

To check the relation between losses of the leastloaded class and fixation events (Charlesworth \& Charlesworth, 1997), a number of multi-locus stochastic simulations were performed in which the fate of each mutation at every locus was followed, and the fixation events counted.

\section{Results}

(i) The rate of accumulation by the ratchet with background selection

(a) Region of parameter space for which $n_{00} \gg 1$. As discussed above, if the rate at which strong mutations occur $\left(u_{\mathrm{b}}\right)$ is not too large, their effect is not too small and $N$ is large (B. Charlesworth et al., 1993), they will stay near their expected equilibrium frequency, and the rate of accumulation of the mildly deleterious is the same as in a population whose size is reduced by the factor $f_{0 \mathrm{~b}}$. In Figs 1 and 2 we plot the average time to successive losses of the least-loaded class measured in the simulations, with a population subject to mutations with two types of effect (T2-class model) and for the case where we have a population of reduced size, $N f_{0 \mathrm{~b}}$, subject to mutations of just one type of effect $\left(s=s_{\mathrm{s}}\right)$, occurring at a rate $u_{\mathrm{s}}$ (T1-class model). We also plot the theoretical prediction based on equation (3) (Theoretical). In Fig. 1 the time between clicks is shown as an increasing function of the population size $(N)$, and in Fig. 2 as a function of the effects of the weaker mutations $\left(s_{\mathrm{s}}\right)$ in a population of $10^{5}$ individuals. For the case when $s_{\mathrm{s}}=0.002$, the

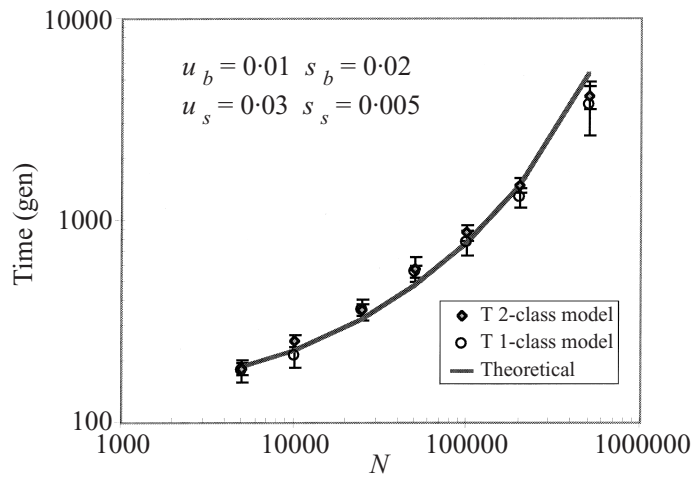

Fig. 1. The mean time between clicks of the ratchet as a function of the population size $N$ in the presence of background selection (T2-class model). The mutation rate for mildly deleterious mutations with selection coefficient $s_{\mathrm{s}}=0.005$ is $u_{\mathrm{s}}=0.03$, and the rate for strongly deleterious mutations with selection coefficient $s_{\mathrm{b}}=0.02$ is $u_{\mathrm{b}}=0 \cdot 01$. The T1-class model is the mean time from simulations with populations of size $f_{0 \mathrm{~b}} N$

$=N \exp \left(-u_{\mathrm{b}} / s_{\mathrm{b}}\right)$, mutation rate $u_{\mathrm{s}}$ and selection coefficient $s_{\mathrm{s}}$. 'Theoretical' is the time calculated with equation (3).

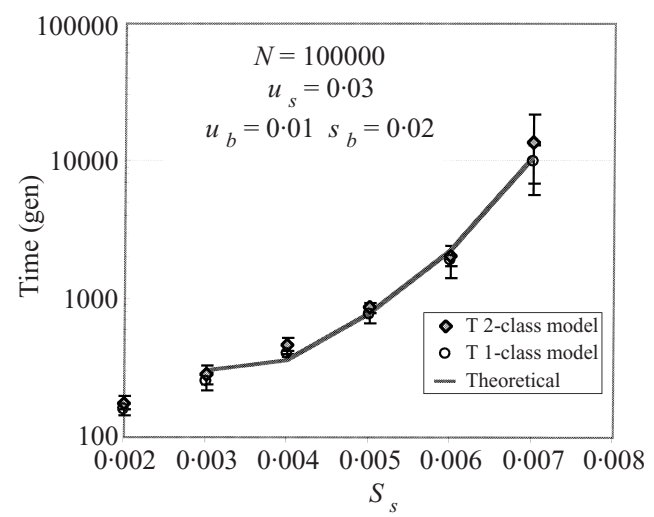

Fig. 2. The average time for a click of the ratchet, as a function of the effect of mildly deleterious mutations, in a population of $10^{5}$ individuals. $s_{\mathrm{b}}=0 \cdot 02, u_{\mathrm{b}}=0.01$ and $u_{\mathrm{s}}$ $=0 \cdot 03$. Other symbols are as in Fig. 1 . For $s_{\mathrm{s}}=0 \cdot 002, n_{00}$ is less than 1 and the diffusion approximation is invalid.

theoretical approximation is invalid because $n_{00}<1$, and another expression should be used to predict the rate of mutation accumulation (Gessler, 1995), as described below. Figs 1 and 2 mostly display cases where the expected size of the least-loaded class in an effectively infinite population, $n_{00}$, is greater than 1 . It is clear that, under this condition, the rate of accumulation of deleterious mutations is increased when compared with the case of occurrence of mutations with just one effect, due to the reduction in $N_{\mathrm{e}}$ and that it can be predicted with reasonable accuracy using equation (3). Several other parameters were simulated that support this conclusion (results not shown). Therefore, even large populations can suffer from a very rapid accumulation of mildly deleterious mutations if the reduction in $N_{\mathrm{e}}$ due to 
Table 1. The time between clicks of Muller's ratchet $(T)$ in the absence and presence of background selection

\begin{tabular}{|c|c|c|c|c|c|c|}
\hline \multirow[b]{3}{*}{$N$} & \multirow{2}{*}{\multicolumn{2}{|c|}{$\begin{array}{l}\text { Absence of background } \\
\text { selection } \\
u_{\mathrm{s}}=0 \cdot 03, s_{\mathrm{s}}=0 \cdot 005\end{array}$}} & \multicolumn{4}{|c|}{$\begin{array}{l}\text { Presence of background selection } \\
u_{\mathrm{s}}=0.03, s_{\mathrm{s}}=0.005\end{array}$} \\
\hline & & & \multicolumn{2}{|c|}{$u_{\mathrm{b}}=0.01, s_{\mathrm{b}}=0.02$} & \multicolumn{2}{|c|}{$u_{\mathrm{b}}=0.01, s_{\mathrm{b}}=0 \cdot 01$} \\
\hline & $T$ & $2 \mathrm{SE}$ & $T$ & $2 \mathrm{SE}$ & $T$ & 2SE \\
\hline 5000 & 228 & 12 & 185 & 13 & 182 & 27 \\
\hline 10000 & 324 & 32 & 256 & 18 & 229 & 11 \\
\hline 50000 & 778 & 108 & 579 & 85 & 413 & 26 \\
\hline 100000 & 1144 & 175 & 875 & 80 & 649 & 79 \\
\hline 500000 & 12819 & 5681 & 4114 & 551 & 2875 & 1189 \\
\hline
\end{tabular}

Results on the effect of background selection, for different values of population size $(N)$ and mutation rates to weakly and strongly deleterious mutations, $u_{\mathrm{s}}$ and $u_{\mathrm{b}}$ respectively. The corresponding selection coefficients are $s_{\mathrm{s}}$ and $s_{\mathrm{b}}$. SE is the standard error. Note that no accumulation of the strongly deleterious mutations was observed in the simulations.

strong deleterious mutations is large. To better see the strength of this effect on the speed of the ratchet, Table 1 shows the effect of background selection on the average time between clicks of the ratchet as seen in the simulations. Note that, in all cases in Table 1, no accumulation of strongly deleterious mutations occurs.

Although the rate of accumulation of mildly deleterious mutations in the presence of strong ones is approximately the same as for a population whose size is reduced by the factor $f_{0 \mathrm{~b}}$ and which has the mutation rate $u_{\mathrm{s}}$, we observe that the rate of accumulation under background selection seems to be systematically lower than expected, although the difference is relatively small. For example, in Fig. 2, the time for the T2-class model is 13965 generations but that for the T1-class model is 10074 for $s_{\mathrm{s}}=0.007$. In fact, if one follows the value of the fraction of individuals that are free of strong deleterious mutations, we observe that the average value of this fraction is slightly bigger than the expected value of $f_{0 \mathrm{~b}}$. Therefore, if this is always true, the reduction in effective population size is slightly smaller than that predicted by the deterministic equilibrium value, so the diffusion coefficient in the diffusion approximation is smaller, which will increase the time to absorption.

(b) Region of parameter space for which $n_{00}<1$. In the above analysis, we have quantified the ratchet mechanism as classically defined by Muller (1964, p. 2), i.e. loss of the least-loaded class due to drift after the equilibrium between mutation and selection is reached. This requires $N s_{\mathrm{b}} \gg 1, N s_{\mathrm{s}} \gg 1$ and $n_{00}>1$. When $N s_{\mathrm{b}} \gg 1$ and $N s_{\mathrm{s}} \gg 1$ but $n_{00}<1$, mutation accumulation is a quasi-deterministic process and stochasticity plays a second-order role in driving the ratchet (Gessler, 1995). Under these circumstances,

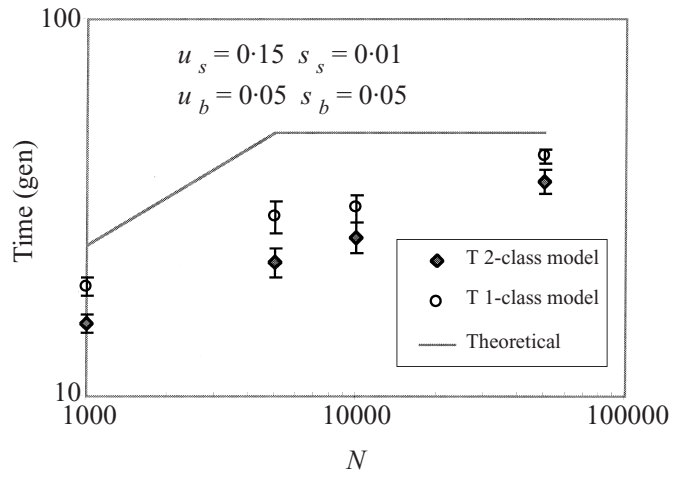

Fig. 3. The average time for a click of the ratchet as a function of the population size for the range of parameter space where $n_{00}<1$. 'Theoretical' is the theoretical value based on equation (8) in Gessler (1995), adjusted for reduced effective population size. Other symbols are as in Fig. 1.

Gessler has proposed an expression for calculating the rate of the ratchet (Gessler, 1995, equation (8)), based on parameterisation of the distribution of the number of mutations in the population. In Figure 3, we show the rate of accumulation for some cases where these conditions are met. Even in these cases, the value for the time is reasonably well predicted by the reduction in population size $f_{0 \mathrm{~b}}$. The theoretical value (Theoretical) obtained with a reduced population size under the approximation of Gessler (1995) seems to predict the rate of mutation accumulation quite well, for this region of parameter space.

\section{(ii) Dynamics of very weakly deleterious mutations}

When mutations have extremely weak effects, such that $N s_{\mathrm{s}}$ or $f_{0 \mathrm{~b}} N s_{\mathrm{s}}$ is of the order of 1 , their dynamics are mainly determined by drift. This means that the 
Table 2. Comparison of the rate of fixation of weak deleterious mutations due to drift $\left(R_{\mathrm{fix}}\right)$ with the rate of the ratchet $\left(R_{\mathrm{rat}}\right)$

\begin{tabular}{|c|c|c|c|c|c|c|c|c|c|}
\hline$N$ & $u_{\mathrm{b}}$ & $S_{\mathrm{b}}$ & $u_{\mathrm{s}}$ & $s_{\mathrm{s}}$ & $n_{00}$ & $N s_{\mathrm{s}}$ & $f_{0 \mathrm{~b}} N s_{\mathrm{s}}$ & $R_{\text {rat }}$ & $R_{\text {fix }}$ \\
\hline \multirow[t]{4}{*}{1000} & $0 \cdot 05$ & $0 \cdot 05$ & $0 \cdot 05$ & $0 \cdot 0025$ & $7 \cdot 6 \times 10^{-7}$ & $2 \cdot 5$ & $0 \cdot 92$ & $0 \cdot 03$ & $0 \cdot 02$ \\
\hline & & 0.05 & & 0.005 & $1.7 \times 10^{-2}$ & 5 & $1 \cdot 84$ & $0 \cdot 02$ & 0.005 \\
\hline & & $0 \cdot 05$ & & $0 \cdot 01$ & $2 \cdot 5$ & 10 & $3 \cdot 68$ & $0 \cdot 015$ & $0 \cdot 0002$ \\
\hline & & $0 \cdot 05$ & & $0 \cdot 02$ & 30 & 20 & $7 \cdot 36$ & 0.005 & $3 \times 10^{-7}$ \\
\hline \multirow[t]{3}{*}{40000} & 0.028 & $0 \cdot 01$ & $0 \cdot 042$ & $0 \cdot 0001$ & $9 \cdot 6 \times 10^{-180}$ & 4 & $0 \cdot 24$ & 0.035 & $0 \cdot 033$ \\
\hline & & $0 \cdot 01$ & & $0 \cdot 0002$ & $1 \cdot 5 \times 10^{-88}$ & 8 & $0 \cdot 49$ & $0 \cdot 03$ & $0 \cdot 02$ \\
\hline & & $0 \cdot 01$ & & $0 \cdot 0005$ & $8 \cdot 0 \times 10^{-34}$ & 20 & $1 \cdot 22$ & $0 \cdot 03$ & $0 \cdot 01$ \\
\hline \multirow[t]{4}{*}{100000} & 0.035 & $0 \cdot 005$ & $0 \cdot 015$ & $0 \cdot 0001$ & $6.5 \times 10^{-64}$ & 10 & $0 \cdot 01$ & $0 \cdot 014$ & $0 \cdot 015$ \\
\hline & & $0 \cdot 006$ & & $0 \cdot 0001$ & $2 \cdot 1 \times 10^{-63}$ & 10 & $0 \cdot 03$ & $0 \cdot 014$ & $0 \cdot 015$ \\
\hline & & $0 \cdot 01$ & & $0 \cdot 0001$ & $2 \cdot 2 \times 10^{-62}$ & 10 & $0 \cdot 30$ & $0 \cdot 012$ & $0 \cdot 011$ \\
\hline & & $0 \cdot 03$ & & $0 \cdot 0001$ & $2 \cdot 2 \times 10^{-61}$ & 10 & $3 \cdot 11$ & $0 \cdot 01$ & $0 \cdot 0002$ \\
\hline
\end{tabular}

The rate of fixation is calculated using the standard single-locus formula for the rate of fixation of deleterious mutations: $R_{\mathrm{fix}}=f_{0 \mathrm{~b}} N u_{\mathrm{s}}\left(\exp \left(2 s_{\mathrm{s}}\right)-1\right) /\left(\exp \left(2 f_{0 \mathrm{~b}} N s_{\mathrm{s}}\right)-1\right)$ (Crow \& Kimura, 1970, chap. 8). $n_{00}$ is the equilibrium size of the leastloaded class. Other parameters are as in Table 1.

frequencies of these mutations can reach high values, and the assumption of negligible back-mutation becomes unrealistic. This implies that the most important component of the ratchet mechanism, its irreversibility, is likely to be lost in real biological systems. Studies of completely linked sets of weakly selected loci with reversible mutation have been done by Li (1987), Comeron et al. (1999) and McVean \& Charlesworth (2000).

One point to note is the fact that, when $f_{0 \mathrm{~b}} N s_{\mathrm{s}} \gg 1$, the rate of the ratchet is much bigger than the rate of fixation of deleterious mutations due to drift calculated from the standard single-locus formula (Crow \& Kimura, 1970, chap. 8; Higgs \& Woodcock, 1995). For a given $N$ and $s$, the rate of fixation due to drift increases linearly with $u$, but the rate of the ratchet increases in an exponential fashion (D. Charlesworth et al., 1993; Gordo \& Charlesworth, 2000a, b). This behaviour of the ratchet is not seen when $N s_{\mathrm{s}}$ or $f_{0 \mathrm{~b}} N s_{\mathrm{s}}<1$, as shown in Table 2. Clearly, when $f_{0 \mathrm{~b}} N s_{\mathrm{s}}>1$ the rate of the ratchet is greater than the rate of fixation due to drift, but, as this quantity tends to or becomes smaller than 1, the rate of the ratchet and the single-locus rate of fixation converge to the same value, approaching the mutation rate $u_{\mathrm{s}}$ from below as $f_{0 \mathrm{~b}} N s_{\mathrm{s}} \rightarrow 0$. For $f_{0 \mathrm{~b}} N s_{\mathrm{s}} \ll 1$, this means that mutation accumulation is mainly occurring due to independent fixations of deleterious mutations at different loci. It is therefore not correct to call this a ratchet, since there is no acceleration of the rate of fixation over that expected with independence between loci. In all the cases shown in Table $2, N s_{\mathrm{s}} \gg 1$, so that the rate of fixation due to drift of the small-effect mutations would be negligible if they occurred in isolation. It is the effect of background selection that allows them to experience an accelerated rate of fixation (Charlesworth, 1996; Charlesworth \& Charlesworth, 1998). Even in the presence of a ratchet

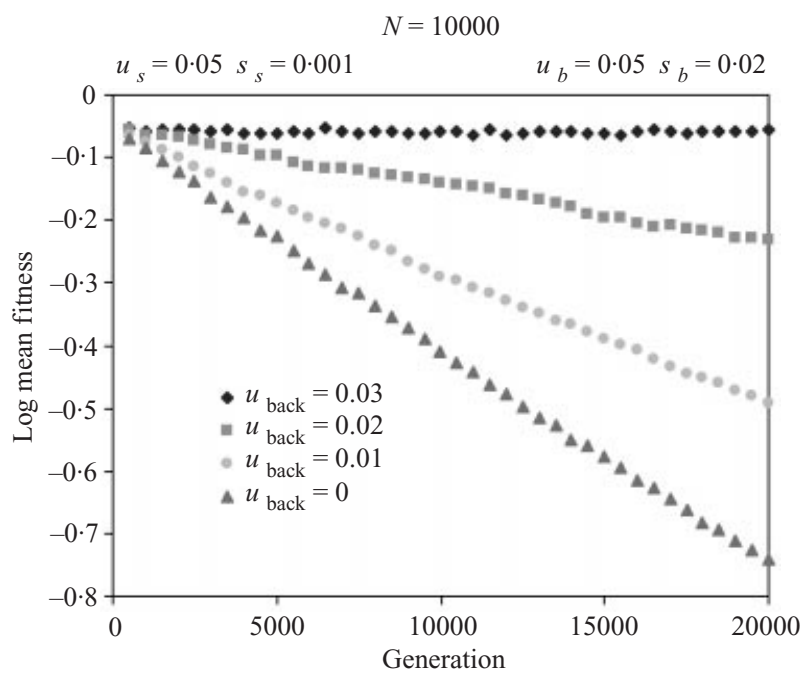

Fig. 4. The change in mean fitness over time with reversible mutation occurring at a rate $u_{\text {back }}$ per individual per generation.

for the large-effect mutations, for the cases where $N$ $=10^{5}$ and $s_{\mathrm{b}}=0.005$ or 0.006 in Table 2 , the rate of fixation of the small-effect mutations seems to be well predicted by a simple reduction in $N_{\mathrm{e}}$ by a factor of $f_{0 \mathrm{~b}}$. This is because the distribution of the large-effect mutations is close to the deterministic equilibrium expectation when $N$ is large (Charlesworth \& Charlesworth, 1997), so that the size of the class free of such mutations stays close to $f_{0 \mathrm{~b}} N$ over long periods of time (as observed in the simulations).

In Fig. 4, the effect of back-mutation is considered for cases where $f_{0 \mathrm{~b}} N s_{\mathrm{s}}<1$. As is intuitively obvious, the bigger the back-mutation rate $\left(u_{\text {back }}\right)$, the smaller the decline in log mean fitness. Note that, when there is back-mutation, we can no longer talk about a ratchet because, even if the least-loaded class is lost at some point in time, this loss is not irreversible, since it 
Table 3. The relation between a mutation model with two types of mutational effects and a model with a constant mutation effect equal to the harmonic mean of the two effects $\left(s_{\mathrm{H}}\right)$

\begin{tabular}{|c|c|c|c|c|c|c|c|c|c|c|}
\hline$N$ & $u_{\mathrm{s}}$ & $s_{\mathrm{s}}$ & $u_{\mathrm{b}}$ & $s_{\mathrm{b}}$ & $s_{\mathrm{H}}$ & $T\left(s_{\mathrm{s}}\right)$ & $T\left(s_{\mathrm{b}}\right)$ & $T\left(s_{\mathrm{H}}\right)$ & $\Delta \ln \bar{w}$ & $\Delta \ln \bar{w}_{s_{\mathrm{H}}}$ \\
\hline \multirow[t]{6}{*}{500} & $0 \cdot 05$ & $0 \cdot 02$ & 0.05 & $0 \cdot 03$ & $0 \cdot 024$ & 90 & 222 & 70 & $-3 \cdot 6 \times 10^{-4}$ & $-3.4 \times 10^{-4}$ \\
\hline & 0.05 & $0 \cdot 01$ & 0.05 & 0.03 & 0.015 & 52 & 379 & 40 & $-2 \cdot 7 \times 10^{-4}$ & $-3 \cdot 8 \times 10^{-4}$ \\
\hline & $0 \cdot 05$ & $0 \cdot 01$ & 0.05 & $0 \cdot 015$ & $0 \cdot 012$ & 57 & 92 & 33 & $-3 \cdot 4 \times 10^{-4}$ & $-3.6 \times 10^{-4}$ \\
\hline & $0 \cdot 05$ & $0 \cdot 0075$ & $0 \cdot 05$ & $0 \cdot 015$ & $0 \cdot 01$ & 44 & 102 & 32 & $-3 \cdot 2 \times 10^{-4}$ & $-3 \cdot 1 \times 10^{-4}$ \\
\hline & $0 \cdot 05$ & $0 \cdot 01$ & 0.075 & $0 \cdot 015$ & $0 \cdot 0125$ & 50 & 55 & 25 & $-4 \cdot 7 \times 10^{-4}$ & $-4.9 \times 10^{-4}$ \\
\hline & 0.025 & $0 \cdot 02$ & 0.075 & 0.03 & 0.0267 & 164 & 145 & 85 & $-3 \cdot 3 \times 10^{-4}$ & $-3 \cdot 4 \times 10^{-4}$ \\
\hline \multirow[t]{6}{*}{1000} & 0.05 & $0 \cdot 02$ & 0.05 & 0.03 & 0.024 & 141 & 389 & 97 & $-2 \cdot 2 \times 10^{-4}$ & $-2.5 \times 10^{-4}$ \\
\hline & $0 \cdot 05$ & $0 \cdot 01$ & $0 \cdot 05$ & $0 \cdot 03$ & $0 \cdot 015$ & 57 & 898 & 49 & $-2 \cdot 1 \times 10^{-4}$ & $-3 \cdot 1 \times 10^{-4}$ \\
\hline & 0.05 & 0.0075 & 0.05 & $0 \cdot 03$ & 0.012 & 46 & 1199 & 47 & $-1.9 \times 10^{-4}$ & $-2 \cdot 6 \times 10^{-4}$ \\
\hline & $0 \cdot 05$ & $0 \cdot 01$ & $0 \cdot 05$ & $0 \cdot 015$ & $0 \cdot 012$ & 64 & 121 & 40 & $-2 \cdot 8 \times 10^{-4}$ & $-2 \cdot 6 \times 10^{-4}$ \\
\hline & $0 \cdot 05$ & $0 \cdot 01$ & 0.075 & $0 \cdot 015$ & $0 \cdot 0125$ & 58 & 69 & 31 & $-3.9 \times 10^{-4}$ & $-4.0 \times 10^{-4}$ \\
\hline & 0.025 & 0.02 & 0.075 & 0.03 & $0 \cdot 0267$ & 252 & 277 & 152 & $-1.9 \times 10^{-4}$ & $-1.8 \times 10^{-4}$ \\
\hline
\end{tabular}

$T\left(s_{\mathrm{s}}\right)$ and $T\left(s_{\mathrm{b}}\right)$ are the times to loss of the least-loaded class of mutations with selection coefficient $s_{\mathrm{s}}$ and $s_{\mathrm{b}}$, respectively. $T\left(s_{\mathrm{H}}\right)$ is the time for a click of the ratchet in a population subject to mutations with fitness effect $s_{\mathrm{H}} . \Delta \ln \bar{w}$ and $\Delta \ln \bar{w}_{s_{\mathrm{H}}}$ are the rates of decline in mean fitness for each mutational model. Other parameters are as in Table 1.

can be rebuilt, and an equilibrium is eventually established even with no recombination (McVean \& Charlesworth, 2000).

\section{(iii) Accumulation of both types of mutations}

In addition to the accumulation of the small-effect mutations, stochastic loss of the least-loaded class of the large-effect mutations may also occur. This is especially likely in very small populations, when the mutation rate to the large-effect mutations is large or when the difference in the selection coefficients is not very large. In Table 3 we show the results of simulations for some cases where this occurs. We also show the rate of decline in log mean fitness per generation due to the accumulation of both types of mutations $(\Delta \ln \bar{w})$. We then compare this decline with the one occurring in a population subject to mutations whose fitness effect is equal to the harmonic mean $\left(s_{\mathrm{H}}\right)$ of the fitness effects of the corresponding two-type mutation model:

$\frac{1}{s_{\mathrm{H}}}=\frac{1}{\left(u_{\mathrm{b}}+u_{\mathrm{s}}\right)}\left(\frac{u_{\mathrm{b}}}{s_{\mathrm{b}}}+\frac{u_{\mathrm{s}}}{s_{\mathrm{s}}}\right)$.

This reflects the fact that the mean number of mutations, and the deterministic equilibrium size of the least-loaded class, are the same in both cases.

We find that, in these small populations, when the rates of accumulation of each type of mutation are of the same order of magnitude, the rate of decline in log mean fitness seems to be well approximated using the harmonic mean of the selection coefficients.

If we equate the decline in $\log$ mean fitness in the two cases we will have

$-\left[\frac{s_{\mathrm{s}}}{T\left(s_{\mathrm{s}}\right)}+\frac{s_{\mathrm{b}}}{T\left(s_{\mathrm{b}}\right)}\right] \approx-\left[\frac{s_{\mathrm{H}}}{T\left(s_{\mathrm{H}}\right)}\right]$ where $T\left(s_{\mathrm{s}}\right)$ and $T\left(s_{\mathrm{b}}\right)$ are the times to loss of the leastloaded class for each mutation type.

Let $K_{\mathrm{s}}$ and $K_{\mathrm{b}}$ be the probabilities that a click of the ratchet involves the class of small- and large-effect mutations, respectively. We can rewrite the above expression as follows:

$$
\begin{aligned}
& -\left[s_{\mathrm{s}} \frac{K_{\mathrm{s}}}{T\left(s_{\mathrm{H}}\right)}+s_{\mathrm{b}} \frac{K_{\mathrm{b}}}{T\left(s_{\mathrm{H}}\right)}\right] \\
& \approx-\frac{1}{\left(u_{\mathrm{s}} / s_{\mathrm{s}}\right)+\left(u_{\mathrm{b}} / s_{\mathrm{b}}\right)}\left[\frac{u_{\mathrm{s}}}{T\left(s_{\mathrm{H}}\right)}+\frac{u_{\mathrm{b}}}{T\left(s_{\mathrm{H}}\right)}\right] .
\end{aligned}
$$

Then we can, tentatively, approximate the probabilities by:

$K_{\mathrm{s}} \approx \frac{u_{\mathrm{s}} / s_{\mathrm{s}}}{\left(u_{\mathrm{s}} / s_{\mathrm{s}}\right)+\left(u_{\mathrm{b}} / s_{\mathrm{b}}\right)}$

and

$K_{\mathrm{b}} \approx \frac{u_{\mathrm{b}} / s_{\mathrm{b}}}{\left(u_{\mathrm{s}} / s_{\mathrm{s}}\right)+\left(u_{\mathrm{b}} / s_{\mathrm{b}}\right)}$

i.e. the proportion of the mean number of mutations of each class.

Since $T\left(s_{\mathrm{H}}\right)$ can be calculated, with these expressions we can calculate the rate of movement for each class. As illustrated in Table 4, this approximation gives accurate results only in cases where the mutation rates and selection coefficients for each class are fairly similar.

From many different parameters values tested, for which accumulation of both mutations occurs, we draw the following conclusion: as long as the rates of movement of each class are of the same order of magnitude, the decline in log mean fitness seems to be well approximated using the harmonic mean of the selection coefficients. When this is true, and when the mutation rates and selection coefficients for each class 
Table 4. The times to loss of each class when there is accumulation of both types of mutations

\begin{tabular}{|c|c|c|c|c|c|c|c|c|c|}
\hline$u_{\mathrm{s}}$ & $s_{\mathrm{s}}$ & $u_{\mathrm{b}}$ & $s_{\mathrm{b}}$ & $T\left(s_{\mathrm{s}}\right)$ & $T\left(s_{\mathrm{b}}\right)$ & $\begin{array}{l}T\left(s_{\mathrm{s}}\right) \\
\text { predicted }\end{array}$ & $\begin{array}{l}T\left(s_{\mathrm{b}}\right) \\
\text { predicted }\end{array}$ & $\Delta \ln \bar{w}$ & $\Delta \ln \bar{w}_{s_{\mathrm{H}}}$ \\
\hline 0.04 & $0 \cdot 001$ & $0 \cdot 04$ & $0 \cdot 01$ & 34 & 835 & 34 & 341 & $4 \times 10^{-5}$ & $6 \times 10^{-5}$ \\
\hline $0 \cdot 04$ & 0.002 & $0 \cdot 04$ & $0 \cdot 01$ & 43 & 455 & 47 & 234 & $7 \times 10^{-5}$ & $8 \times 10^{-5}$ \\
\hline $0 \cdot 04$ & 0.005 & $0 \cdot 04$ & $0 \cdot 01$ & 90 & 309 & 100 & 201 & $9 \times 10^{-5}$ & $1 \times 10^{-4}$ \\
\hline $0 \cdot 04$ & $0 \cdot 009$ & 0.04 & $0 \cdot 01$ & 181 & 228 & 205 & 228 & $9 \times 10^{-5}$ & $9 \times 10^{-5}$ \\
\hline $0 \cdot 04$ & $0 \cdot 005$ & $0 \cdot 06$ & $0 \cdot 0075$ & 93 & 86 & 88 & 88 & $1 \times 10^{-4}$ & $1 \times 10^{-4}$ \\
\hline $0 \cdot 02$ & 0.005 & $0 \cdot 04$ & 0.008 & 268 & 234 & 236 & 189 & $5 \times 10^{-5}$ & $6 \times 10^{-5}$ \\
\hline $0 \cdot 01$ & $0 \cdot 0025$ & $0 \cdot 02$ & $0 \cdot 008$ & 328 & 2699 & 483 & 772 & $1 \times 10^{-5}$ & $2 \times 10^{-5}$ \\
\hline
\end{tabular}

The population size is 10000 . The predicted times are calculated using equations (4a) and (4b). Other parameters are as in Table 3.

of mutations are similar, the rate of movement of each class seems to be reasonably well approximated using equations (4a) and (4b). When one of the mutational classes starts to accumulate at a rate that is an order of magnitude lower than the other, and $s_{\mathrm{b}} \gg s_{\mathrm{s}}$, one can use the reduction in effective population approximation to predict the rate of movement of the latter, and approximate the rate of movement of the large-effect deleterious class by treating it as though the small-effect mutations were absent.

\section{Discussion}

The accumulation of deleterious mutations in finite non-recombining populations has received a considerable amount of attention, because of its potential importance for the degeneration of $\mathrm{Y}$ chromosomes, the extinction of asexual populations and the evolution of recombination (Muller, 1964; Felsenstein, 1974; Haigh, 1978; Maynard Smith, 1978; Pamilo et al., 1987; Rice, 1987; Lynch \& Gabriel, 1990; Gessler, 1995; Higgs \& Woodcock, 1995; Charlesworth \& Charlesworth, 1997; Gessler \& Xu, 1999). For mathematical simplicity, the quantification of this process is usually done under the assumption of equal mutation effects across loci. Although there have been some studies in which a continuous distribution of mutation effects was considered (Butcher, 1995; Gessler, 1995), analytical expressions for the rate of this process exist only for cases of constant selection coefficients.

\section{(i) The rate of the ratchet under background selection}

Recent studies on the rate of occurrence of deleterious mutations and on the values of the selection coefficients of those mutations have cast doubts on the simple assumption that all detrimental mutations have similar deleterious effects (Keightley, 1996; Davies et al., 1999). Davies et al. (1999) have studied the effects of induced mutations in Caenorhabditis elegans and observed that the distribution of mutation effects appears to be bimodal. A simple discrete distribution with two classes of mutation effects seems to fit the data better than a continuous gamma distribution. While mutations with mean heterozygous fitness effects of the order of $1-2 \%$ have been inferred from laboratory experiments on Drosophila melanogaster (Charlesworth \& Hughes, 2000), there may well exist a numerous class of mutations with much smaller effects (Davies et al., 1999) which are simply very difficult to detect in classic mutation accumulation experiments. We have tried to quantify the process of mutation accumulation by Muller's ratchet in these circumstances

With a simple two-category distribution of mutation effects $\left(s_{\mathrm{b}}\right.$ and $\left.s_{\mathrm{s}}\right)$, in which we have a strong asymmetry in mutation rates $\left(u_{\mathrm{b}}<u_{\mathrm{s}}\right)$, namely those mutations that are more deleterious $\left(s_{\mathrm{b}}\right)$ occur at a lower rate than those that are less deleterious $\left(s_{\mathrm{s}}\right)$, we found that the effect of the strong mutations, which do not accumulate, on the rate of accumulation of the weaker ones can be generally well predicted, in the absence of recombination, by the background selection principle with the appropriate reduction in effective population size (Fisher, 1930; B. Charlesworth et al., 1993).

As with the equal-effects mutation model, we can use an explicit expression to quantify the rate of the ratchet, under conditions when the large-effect mutations do not accumulate.

\section{(ii) The rate of the ratchet in small populations}

In sufficiently small populations, both large- and small-effect mutations are likely to accumulate at high rates. In these circumstances, we have compared the decline in log mean fitness of the population with the decline in log mean fitness of a population subject to mutations whose effect $s_{\mathrm{H}}$ is given by the harmonic mean of the selection coefficients of the two classes of mutations (Gessler, 1995). If both mutations accumulate at similar rates, then the rate of decline in $\log$ mean fitness is well approximated by the harmonic mean formula (as shown in Table 4). But if one of the 
mutation types accumulates at a much lower rate than the other, then the background selection effect becomes evident, and this approximation is no longer appropriate (as shown in Tables 3 and 4).

In a haploid asexual population subject to deleterious mutations of equal effects, it is known that there is a one-to-one correspondence between the loss of the least-loaded classes and the fixation of deleterious mutations in the entire population (Higgs \& Woodcock, 1995; Charlesworth \& Charlesworth, 1997; Bergstrom \& Pritchard, 1998). In order to check the rate of advance of the ratchet with the rate of fixation events in the two-type mutation model, we ran multi-locus simulations where the fate of each mutation was followed. We observed that fixation events follow clicks of the ratchet, as previously thought.

\section{(iii) $Y$ and neo- $Y$ chromosomes}

Muller's ratchet and/or background selection may have played a role in sex chromosome differentiation in animals (Charlesworth, 1978, 1996) and plants (Filatov et al., 1999), in which sex is determined by a system of $\mathrm{X}$ and $\mathrm{Y}$ chromosomes. Systems like this have evolved repeatedly and independently throughout evolutionary history. Sex chromosomes are thought to have evolved from a set of homologous, proto-X and proto-Y chromosomes, which stopped recombining completely or over a very large region. For example, it has been postulated recently, that the human Y chromosome is the result of at least four inversions that prevented recombination over large regions of the X and Y (Lahn \& Page, 1999). The Y chromosome of the plant Silene latifolia, and the neoY chromosome of Drosophila miranda, which results from a fusion between a chromosome arm and the old $\mathrm{Y}$ chromosome, are much more recent than this (Filatov et al., 1999; Bachtrog \& Charlesworth, 2000; Yi \& Charlesworth, 2000). The same principles that apply to a primeval non-recombining Y chromosome also apply to a neo-Y chromosome of Drosophila.

While estimates of the deleterious mutation rate and selection coefficients against these mutations exist from various studies on mutation accumulation in Drosophila, the values of these parameters for mammals and plants are not so well known (Keightley \& Eyre-Walker, 1999). This makes the quantification of the combined role of Muller's ratchet and background selection somewhat difficult. Tentatively, we can ask about the approximate time scale at which these two processes are expected to occur in these different organisms.

The effective population size of $D$. miranda lies between $10^{5}$ and $10^{6}$ (Yi \& Charlesworth, 2000; Bachtrog \& Charlesworth, 2000). Its neo-Y chromosome constitutes about one-fifth of the genome.
Estimates of the haploid genomic deleterious mutation rate in Drosophila vary between 0.5 to 0.05 (Keightley \& Eyre-Walker, 1999, table 1). Assume, for the sake of argument, that the mutation rate for the neo-Y to strongly deleterious mutations, which can be detected in mutation accumulation experiments (i.e. with a heterozygous selection coefficient of $1-2 \%$ ), is $0 \cdot 01$. The haploid effective population size after correction due to background selection exerted by the strong mutations will then lie between $2 \times 10^{5}$ and $3 \times 10^{5}$ (taking account of the fact that the number of breeding males is half the population). Every mutation whose effect is $<0.5 \times 10^{-5}$ will be effectively neutral in the presence of these strongly deleterious mutations and will experience an accelerated rate of fixation (Charlesworth, 1996). For mutations whose effect is bigger than this, the ratchet mechanism will take place under background selection. In Table 5, we quantify the expected rate of the ratchet in the presence of background selection for mutations with different selection coefficients. This quantification is done, as explained previously, using equation (3) when $n_{00}>1$, and equation (8) of Gessler (1995) when $n_{00}<1$. We also show the time scale of this process for the $\mathrm{Y}$ chromosome of the plant Silene latifolia, assuming an effective population size of around $10^{6}$ (Filatov et al., 1999). The values for the $Y$ chromosome deleterious mutation rate assumed here are about one-twelfth the haploid deleterious mutation rate estimates from some species of selfing plants (Drake et al., 1998). This is done using the number of chromosomes in Silene latifolia as a very rough guide, despite the fact that the $\mathrm{X}$ is bigger than the autosomes and the $\mathrm{Y}$ bigger than the X (Matsunaga et al., 1999). The large selection coefficients considered here are lower than the estimated values from the mutation accumulation experiments in Arabidopsis thaliana (Schultz et al., 1999), since these are probably overestimates.

The effective population size of mammals is probably about an order of magnitude smaller than that of Drosophila or Silene (Charlesworth, 1996). Given that the deleterious mutation rate, at least in humans, is very high (Eyre-Walker \& Keightley, 1999; Nachman \& Crowell, 2000), if the first inversion on the mammalian $\mathrm{Y}$ chromosome caused a region of about half its size to stop recombining, then the reduction in effective population size due to background selection might have caused a rapid accumulation and fixation of mildly deleterious mutations due to the ratchet. Given that the $\mathrm{X}$ chromosome of eutherian mammals represents $3-5 \%$ of the haploid genome (Graves, 1995; Deloukas et al., 1998), a deleterious mutation rate for the proto-Y of 0.04 can be considered reasonable, from current estimates for the deleterious mutation rate in hominids (Eyre-Walker \& Keightley, 1999). In Table 5, we assume this value for the mutation rate per mammalian 
Table 5. Mean fitness and expected number of fixations after 1 million generations

\begin{tabular}{|c|c|c|c|c|c|c|c|c|}
\hline$N$ & $u_{\mathrm{s}}$ & $s_{\mathrm{s}}$ & $u_{\mathrm{b}}$ & $s_{\mathrm{b}}$ & $n_{00}$ & $T$ (generations) & $\bar{w} / \bar{w}_{i}$ & $\begin{array}{l}\text { No. of } \\
\text { fixations }\end{array}$ \\
\hline \multicolumn{9}{|c|}{ Drosophila miranda neo-Y chromosome } \\
\hline \multirow[t]{6}{*}{125000} & $0 \cdot 024$ & $0 \cdot 005$ & $0 \cdot 008$ & $0 \cdot 02$ & 690 & $4508^{a}(10446)$ & $0 \cdot 31(0 \cdot 62)$ & $222(96)$ \\
\hline & & $0 \cdot 001$ & & 0.02 & $3 \times 10^{-6}$ & $250^{b}(333)$ & $0 \cdot 018(0 \cdot 05)$ & 4000 (3003) \\
\hline & & $0 \cdot 005$ & & $0 \cdot 01$ & 462 & $2440^{a}(10446)$ & $0 \cdot 13(0.62)$ & $410(96)$ \\
\hline & & $0 \cdot 001$ & & $0 \cdot 01$ & $2 \times 10^{-6}$ & $250^{b}(333)$ & $0 \cdot 018(0 \cdot 05)$ & $4000(3003)$ \\
\hline & & $0 \cdot 0005$ & & $0 \cdot 01$ & $8 \times 10^{-17}$ & $133^{b}(133)$ & $0 \cdot 023(0 \cdot 023)$ & 7519 (7519) \\
\hline & & $0 \cdot 005$ & $0 \cdot 016$ & $0 \cdot 02$ & 462 & $2440^{a}(10446)$ & $0 \cdot 13(0 \cdot 62)$ & $410(96)$ \\
\hline \multicolumn{9}{|c|}{ Silene latifolia $\mathrm{Y}$ chromosome } \\
\hline \multirow[t]{4}{*}{500000} & $0 \cdot 0075$ & $0 \cdot 002$ & $0 \cdot 0025$ & $0 \cdot 01$ & 9158 & $3 \times 10^{7 a}\left(6 \times 10^{8}\right)$ & $1 \cdot 00(1 \cdot 00)$ & $0(0)$ \\
\hline & & $0 \cdot 0003$ & & $0 \cdot 01$ & $5 \times 10^{-6}$ & $1111^{b}(1111)$ & $0.76(0 \cdot 76)$ & $900(900)$ \\
\hline & $0 \cdot 015$ & $0 \cdot 002$ & $0 \cdot 005$ & $0 \cdot 02$ & 215 & $1306^{a}(1548)$ & $0 \cdot 22(0 \cdot 27)$ & $766(646)$ \\
\hline & & $0 \cdot 0005$ & & $0 \cdot 02$ & $4 \times 10^{-8}$ & $400^{b}(400)$ & $0 \cdot 29(0 \cdot 29)$ & $2500(2500)$ \\
\hline \multicolumn{9}{|c|}{ Mammalian Y chromosome } \\
\hline \multirow[t]{3}{*}{50000} & $0 \cdot 03$ & $0 \cdot 007$ & $0 \cdot 01$ & $0 \cdot 02$ & 417 & $2442^{a}(6356)$ & $0 \cdot 056(0 \cdot 33)$ & $410(157)$ \\
\hline & & $0 \cdot 005$ & & $0 \cdot 02$ & 75 & $469^{a}(663)$ & $2 \times 10^{-5}\left(5 \times 10^{-4}\right)$ & $2132(1508)$ \\
\hline & & 0.005 & & $0 \cdot 06$ & 105 & $586^{a}(663)$ & $2 \times 10^{-4}\left(5 \times 10^{-4}\right)$ & $1706(1508)$ \\
\hline \multirow[t]{2}{*}{10000} & $0 \cdot 024$ & $0 \cdot 005$ & $0 \cdot 016$ & $0 \cdot 02$ & 37 & $304^{a}(483)$ & $7 \times 10^{-8}\left(3 \times 10^{-5}\right)$ & $3289(2070)$ \\
\hline & & $0 \cdot 0005$ & & $0 \cdot 02$ & $6 \times 10^{-18}$ & $100^{b}(105)$ & $0.007(0.009)$ & $10000(9524)$ \\
\hline
\end{tabular}

The expected time scale of operation of Muller's ratchet under background selection for Y chromosomes and the neo-Y chromosomes of Drosophila miranda. Values calculated with equation (3) are labelled (a) and those with equation (8) in Gessler (1995) corrected for a reduced $N$ are labelled $(b) . \bar{w} / \bar{w}_{i}$ is the ratio between the expected mean fitness after $1 \mathrm{million}$ generations and the initial mean fitness. The values in parentheses are the expected values in the absence of strong mutations.

proto-Y chromosome per generation, and an effective population size one order of magnitude below the one we assume for Drosophila. We show the expected time between clicks of the ratchet for different values of the selection coefficients. We observe that, if mildly deleterious mutations are as frequent as we are assuming, they can accumulate in the presence of strongly deleterious mutations at significant rates and cause a considerable decline in the mean fitness of the population of $Y$ chromosomes in a short evolutionary time. From Table 5, we observe that the effect of background selection in increasing the rate of the ratchet is only important in the cases where $n_{00} \gg 1$.

If, in addition to background selection due to strongly deleterious mutations and Muller's ratchet causing accumulation of mildly deleterious mutations, there is a third class of mutations with effects $s_{\mathrm{w}} \sim 1 / N_{\mathrm{e}}$, we expect these mutations to show an accelerated rate of fixation due to the background selection effects of the more strongly deleterious ones (Charlesworth, 1996).

\section{(v) The accumulation of transposable elements due to Muller's ratchet}

Transposable elements (TEs) are sequences that can insert into new locations of the genome. Because of their property of self-replication, they would invade every site into which they can insert if there were no forces opposing transposition. Excision, deleterious effects of mutations induced by the insertion itself, or selection against chromosomal rearrangements created by ectopic recombination between elements, are among the forces thought to balance transposition (Charlesworth \& Langley, 1989; Charlesworth et al., 1992 a, b, 1994, 1997). These forces prevent TEs from occupying coding regions, and can keep TEs at low frequencies in the sites to which they insert, even outside the coding regions.

The absence of recombination in $\mathrm{Y}$ or neo- $\mathrm{Y}$ chromosomes has the automatic consequence of reducing ectopic exchange and reducing selection against rearrangements. This would lead to the accumulation of TEs in these chromosomes, as observed in various organisms (Steinemann \& Steinemann, 1992, 1998; Wichman et al., 1992). In addition, an increased rate of accumulation of these elements due to the ratchet and/or background selection might be responsible for the numerous copies of TEs present in $\mathrm{Y}$ and neo-Y chromosomes (Charlesworth et al., 1994). We illustrate the time scale of this in the following. Consider the specific case of the neo-Y chromosome of D. miranda, whose age is estimated to be about $1 \mathrm{MY}$ (Bachtrog \& Charlesworth, 2000; Yi \& Charlesworth, 2000). Measures of transposition rate have been described for D. melanogaster, in which a mean transposition rate per haploid euchromatic genome per generation is estimated to be about 0.12 (Maside et al., 2000). Assuming that the rate of transposition in D. miranda is about the same, a rate of 0.015 for insertion of TEs that cause very weak mutational effects on fitness due 


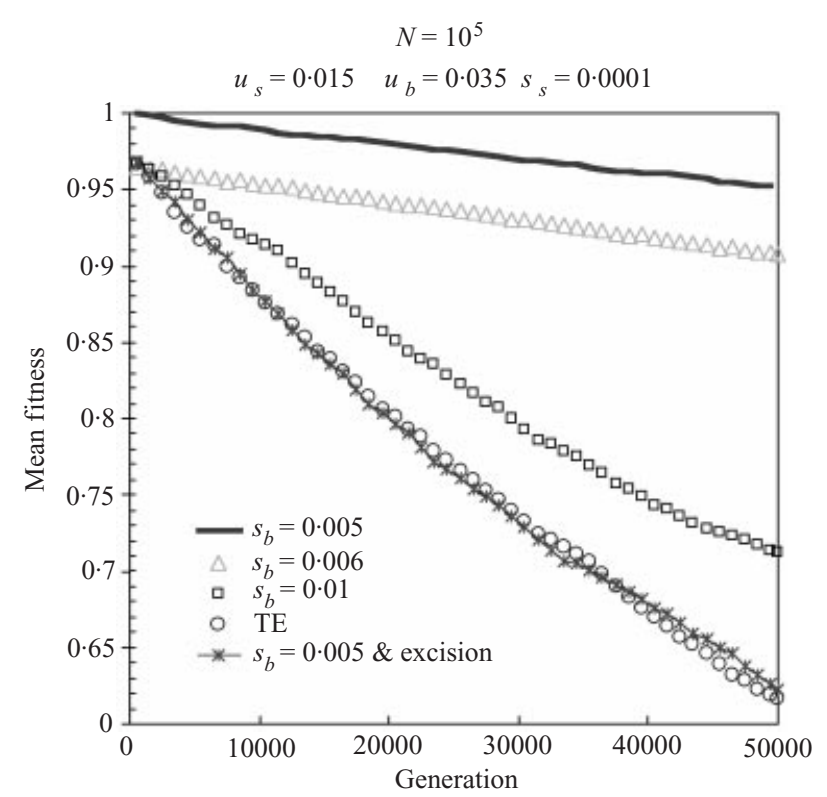

Fig. 5. The accumulation of transposable elements in a hypothetical population of neo-Y chromosomes. The continuous line represents the decline in mean fitness due to TEs in the absence of larger deleterious mutations.

to insertions (for example, into regulatory regions) is possibly a reasonable value for the neo-Y (as assumed in Fig. 5). Multiplicative fitness effects between TEs is also assumed, since synergistic interactions are expected to be weak in the absence of ectopic exchange (Charlesworth et al., 1997).

Suppose that at time zero a fusion that had occurred between the old $\mathrm{Y}$ and an autosomal arm was established, forming a new population of neo-Y chromosomes. In Fig. 5 we show the change in mean fitness over 50000 generations for a population of $10^{5}$ chromosomes, subject to mutations with effect $s_{\mathrm{s}} \sim 10^{-4}$, occurring at rate $0 \cdot 015$. In addition, we also consider the effect of the presence of much largereffect mutations (these can be deleterious point mutations, or strong deleterious mutations caused by TEs when they insert into coding regions). The latter may accumulate by a ratchet, or just cause the background selection effect, depending on the strength of their effect. The population of hypothetical neo-Y chromosomes is initially free of any mutations, so its mean fitness at time 0 has the maximum value 1 (we are ignoring the deleterious mutations that could have got fixed during the fixation of the fusion in the population). As time passes, mutations start to build up and the mean fitness starts to decline. It takes about $1 / s_{\mathrm{s}}$ generations for the mean fitness, with respect to the weakly selected mutations, to reach its equilibrium value $\exp (-u)$ (Johnson, 1999). During this time, successive losses of the least-loaded classes of the weakly deleterious mutations caused by TEs can be observed. Despite this, the mean fitness does not decline much, because the effect of these mutations is extremely small. In the absence of the large-effect mutations, the mean fitness reaches its equilibrium value, $\exp (-0.015)=0.985$, shown as the continuous line in Fig. 5, after approximately 10000 generations, and then decays very slowly. However, over 50000 generations we observe more than 400 clicks of the ratchet for these small-effect mutations.

In the presence of mutations with a selection coefficient of $0 \cdot 01$, the rate of decline in mean fitness after its equilibrium value, 0.951 , has been reached, is slightly bigger than in their absence, and a bigger number of clicks (more than 600) is observed over the same period of time. When the effect of the large-effect mutations drops from $0 \cdot 01$ to $0 \cdot 006-0.005$, then not only does the rate at which TEs accumulate increase, but also a dramatic decline in mean fitness due to the accumulation of the large mutations is observed. Note that, in these circumstances, TEs are accumulating as a result of the accelerated rate of fixation caused by the reduction in effective population size $\left(f_{0 \mathrm{~b}}\right)$ due to the large-effect mutations, even though these mutations actually experience a very slow ratchet (Charlesworth, 1996). If we assume that the larger mutations are accumulating at the same rate as in the absence of TEs (i.e., due to their small fitness effects, TEs do not interfere with the dynamics of the much larger mutations) the predicted value of the mean fitness after 50000 generations is 0.736 , very close to that observed in the simulations $(0 \cdot 712$, for the case where $s_{\mathrm{b}}=0.006$ ). Because over this time the number of TEs has increased from virtually zero to hundreds, and excision was ignored in these simulations, the numbers of TEs will be smaller if excision is considered. But, since the excision rate is thought to be much smaller than the transposition rate (Maside et al., 2000) this effect is likely to be small as we show in Fig. 5 (assuming an excision rate of one-tenth the transposition rate). We therefore expect a very rapid accumulation of TEs by these processes after a neo-Y chromosome is born.

\section{(vi) Conclusions}

We have quantified the rate of accumulation of deleterious mutations by Muller's ratchet considering a model for the distribution of mutation effects that is not only mathematically simple but also seems to be a type of model that fits the data from induced mutation accumulation experiments (Davies et al., 1999). From Table 5 it is clear that mutations with small effects on fitness can accumulate (and fix) at high rates in the presence of mutations with larger effects, if they occur frequently in large chromosomes or chromosome regions that lack recombination. From Fig. 5 we conclude that an accumulation of TEs (with very weak deleterious effects) by a ratchet-like mechanism is expected to occur prior to the accumulation of 
mildly deleterious alleles, although such TEs are assumed to cause a much smaller decline in mean fitness, due to their small effects on fitness.

Muller's ratchet working in combination with background selection can therefore potentially cause a rapid decline in the mean fitness of a large population of $\mathrm{Y}$ or neo-Y chromosomes through the fixation of deleterious alleles, over a biologically reasonable time scale. Of course, the operation of these processes is not incompatible with the operation of other processes: hitchhiking of deleterious mutations due to the fixation of strongly advantageous mutations on the $\mathrm{Y}$ chromosome, accelerated rate of fixation of deleterious mutations with $s<1 / N_{\mathrm{e}}$ on the $\mathrm{Y}$ due to background selection, a lower rate of adaptation of the $\mathrm{Y}$ compared with the $\mathrm{X}$ chromosome, due to the operation of background selection on the $\mathrm{Y}$, and an accelerated rate of fixation of deleterious mutations with $s \sim 1 / N_{\mathrm{e}}$ due to the Hill-Robertson effect (Rice, 1987; Charlesworth, 1996; Orr \& Kim, 1998; McVean \& Charlesworth, 2000; Charlesworth \& Charlesworth, 2000).

I. G. thanks P. Keightley, D. Bachtrog, F. Depaulis and the reviewers for comments on the manuscript. I. G. is supported by the Gulbenkian Foundation and Praxis XXI of Portugal and B.C. by the Royal Society.

\section{References}

Bachtrog, D. \& Charlesworth, B. (2000). Reduced levels of microsatellite variability on the neo-Y chromosome of Drosophila miranda. Current Biology 10, 1025-1031.

Barton, N. H. (1995). Linkage and the limits to natural selection. Genetics 140, 821-841.

Bergstrom, C. T. \& Pritchard, J. (1998). Germline bottlenecks and the evolutionary maintenance of mitochondrial genomes. Genetics 149, 2135-2146.

Butcher, D. (1995). Muller's ratchet, epistasis and mutation effects. Genetics 141, 431-437.

Charlesworth, B. (1978). Model for evolution of Y chromosomes and dosage compensation. Proceedings of the National Academy of Sciences of the USA 75, 5618-5622.

Charlesworth, B. (1994). The effect of background selection against deleterious mutations on weakly selected, linked variants. Genetical Research 63, 213-227.

Charlesworth, B. (1996). The evolution of chromosomal sex determination and dosage compensation. Current Biology 6, 149-162.

Charlesworth, B. \& Charlesworth, D. (1997). Rapid fixation of deleterious alleles can be caused by Muller's ratchet. Genetical Research 70, 63-73.

Charlesworth, B. \& Charlesworth, D. (1998). Some evolutionary consequences of deleterious mutations. Genetica 102-103, 3-19.

Charlesworth, B. \& Charlesworth, D. (2000). The degeneration of Y chromosomes. Philosophical Transactions of Royal Society of London, Series B 355, 1563-1572.

Charlesworth, B. \& Hughes, K. (2000). The maintenance of genetic variation in life-history traits. In Evolutionary Genetics from Molecules to Morphology (ed. R. S. Singh \& C. B. Krimbas), pp. 369-392. Cambridge: Cambridge University Press.
Charlesworth, B. \& Langley, C. H. (1989). The population genetics of Drosophila transposable elements. Annual Reviews in Genetics 23, 251-287.

Charlesworth, B., Lapid, A. \& Canada, D. (1992a). The distribution of transposable elements within and between chromosomes in a population of Drosophila melanogaster. I. Element frequencies and distribution. Genetical Research 60, 103-114.

Charlesworth, B., Lapid, A. \& Canada, D. (1992b). The distribution of transposable elements within and between chromosomes in a population of Drosophila melanogaster. II. Inferences on the nature of selection against elements. Genetical Research 60, 115-130.

Charlesworth, B., Morgan, M. T. \& Charlesworth, D. (1993). The effect of deleterious mutations on neutral molecular variation. Genetics 134, 1289-1303.

Charlesworth, B., Sniegowski, P. \& Stephan, W. (1994). The evolutionary dynamics of repetitive DNA in eukaryotes. Nature 371, 215-220.

Charlesworth, B., Langley, C. H. \& Sniegowski, P. D. (1997). Transposable element distributions in Drosophila. Genetics 147, 1993-1995.

Charlesworth, D., Morgan, M. T. \& Charlesworth, B. (1993). Mutation accumulation in finite outbreeding and inbreeding populations. Genetical Research 61, 39-56.

Comeron, J., Kreitman, M. \& Aguadé, M. (1999). Natural selection on synonymous sites is correlated with gene length and recombination in Drosophila. Genetics 151, 239-249.

Crow, J. F. (1993). Mutation, mean fitness, and genetic load. Oxford Surveys in Evolutionary Biology 9, 3-42.

Crow, J. F. \& Kimura, M. (1970). An Introduction to Population Genetics Theory. Minneapolis, MN: Burgess.

Davies, E. K., Peters, A. D. \& Keightley, P. D. (1999). High frequency of cryptic deleterious mutations in Caenorhabditis elegans. Science 285, 1748-1751.

Deloukas P., et al. (1998). A physical map of 30000 human genes. Science 282, 744-746.

Drake, J. W., Charlesworth, B., Charlesworth, D. \& Crow, J. F. (1998). Rates of spontaneous mutation. Genetics 148, 1667-1686.

Ewens, W. J. (1979). Mathematical Population Genetics. Berlin: Springer.

Eyre-Walker, A. \& Keightley, P. D. (1999). High genomic deleterious mutation rates in hominids. Nature 397, 344-347.

Felsenstein, J. (1974). The evolutionary advantage of recombination. Genetics $\mathbf{7 8}, 737-756$.

Filatov, D., Moneger, F., Negrutiu, I. \& Charlesworth, D. (1999). Low variability in a Y-linked plant gene and its implications for Y-chromosome evolution. Nature 404, 388-390.

Fisher, R. A. (1930). The Genetical Theory of Natural Selection. Oxford: Oxford University Press.

Gessler, D. (1995). The constraints of finite size in asexual population and the rate of the ratchet. Genetical Research 66, 241-253.

Gessler, D. D. \& Xu, S. (1999). On the evolution of recombination and meiosis. Genetical Research 73, 119-131.

Gordo, I. \& Charlesworth, B. (2000a). The degeneration of asexual haploid populations and the speed of Muller's ratchet. Genetics 154, 1379-1387.

Gordo, I. \& Charlesworth, B. (2000b). On the speed of Muller's ratchet. Genetics 156, 2137-2140.

Graves, J. A. (1995). The origin and function of the mammalian Y chromosome and Y-borne genes: an evolving understanding. Bioessays 17, 311-320. 
Haigh, J. (1978). The accumulation of deleterious genes in a population: Muller's ratchet. Theoretical Population Biology 14, 251-267.

Higgs, P. \& Woodcock, G. (1995). The accumulation of mutations in asexual populations and the structure of genealogical trees in the presence of selection. Journal of Mathematical Biology 33, 677-702.

Hill, W. G. \& Robertson, A. (1966). The effect of linkage on limits to artificial selection. Genetical Research 8, 269-294.

Johnson, T. (1999). The approach to mutation-selection balance in an infinite asexual population, and the evolution of mutation rates. Proceedings of the Royal Society of London, Series B 266, 2389-2397.

Keightley, P. D. (1996). Nature of deleterious mutation load in Drosophila. Genetics 144, 1993-1999.

Keightley, P. D. \& Eyre-Walker, A. (1999). Terumi Mukai and the riddle of deleterious mutation rates. Genetics $\mathbf{1 5 3}$, $515-523$.

Kimura, M. \& Maruyama, T. (1966). The mutational load with epistatic gene interactions in fitness. Genetics 54, 1337-1351.

Lahn, B. T. \& Page, D. C. (1999). Four evolutionary strata on the human X chromosome. Science 286, 964-967.

Li, W. (1987). Models of nearly neutral mutations with particular implications for nonrandom usage of synonymous codons. Journal of Molecular Evolution 24, 337-345.

Lynch, M. \& Gabriel, W. (1990). Mutational load and the survival of small populations. Evolution 44, 1725-1737.

Maside, X., Assimacopoulos, S. \& Charlesworth, B. (2000). Rates of movement of transposable elements on the second chromosome of Drosophila melanogaster. Genetical Research 75, 275-284.

Matsunaga, S., Kawano, S., Michimoto, T., Higashiyama, T., Nakao, S., Sakai, A. \& Kuroiwa, T. (1999). Semiautomatic laser beam microdissection of the Y chromosome and analysis of Y chromosome DNA in a dioecious plant, Silene latifolia. Plant Cell Physiology 40, 60-68.

Maynard Smith, J. (1978). The Evolution of Sex. Cambridge: Cambridge University Press.

McVean, G. A. T. \& Charlesworth, B. (2000). The effects of Hill-Robertson interference between weakly selected mutations on patterns of molecular evolution and variation. Genetics 155, 929-944.
Muller, H. (1964). The relation of recombination to mutational advance. Mutational Research 1, 2-9.

Nachman, M. \& Crowell, S. (2000). Estimate of the mutation rate per nucleotide in humans. Genetics 156, 297-304.

Orr, H. A. \& Kim, Y. (1998). An adaptive hypothesis for the evolution of the Y chromosome. Genetics 150, 1693-1698.

Pamilo, P., Nei, M. \& Li, W. (1987). Accumulation of mutations in sexual and asexual populations. Genetical Research 49, 135-146.

Peck, J. R. (1994). A ruby in the rubbish: beneficial mutations, deleterious mutations and the evolution of sex. Genetics 137, 597-606.

Rice, W. R. (1987). Genetic hitchhiking and the evolution of reduced genetic activity of the $\mathrm{Y}$ sex chromosome. Genetics 116, 161-167.

Schultz, S. T., Lynch, M. \& Willis, J. H. (1999). Spontaneous deleterious mutation in Arabidopsis thaliana. Proceedings of the National Academy of Sciences of the USA 96, 11393-11398.

Steinemann, M. \& Steinemann, S. (1992). Degenerating Y chromosome of Drosophila miranda, a trap for retrotransposons. Proceedings of the National Academy of Sciences of the USA 89, 7591-7595.

Steinemann, M. \& Steinemann, S. (1998). Enigma of Y chromosome degeneration: neo- $\mathrm{Y}$ and neo-X chromosomes a model for sex chromosome evolution. Genetica 102/103, 409-420.

Stephan, W., Chao, L. \& Smale, J. G. (1993). The advance of Muller's ratchet in a haploid asexual population: approximate solutions based on diffusion theory. Genetical Research 61, 225-231.

Stephan, W., Charlesworth, B. \& McVean, G. (1999). The effect of background selection at a single locus on weakly selected partially linked variants. Genetical Research 73, 133-146.

Wichman, H. A., Van den Bussche, R. A., Hamilton, M. J. \& Baker, R. J. (1992). Transposable elements and the evolution of genome organization in mammals. Genetica 86, 287-293.

Yi, S. \& Charlesworth, B. (2000). Contrasting patterns of molecular evolution of the genes on the new and old sex chromosomes of Drosophila miranda. Molecular Biology and Evolution 17, 703-717. 